\title{
Atomic mechanisms of grain boundary diffusion: Low versus high temperatures
}

\author{
A. SUZUKI, Y. MISHIN \\ School of Computational Sciences, MSN 5C3, George Mason University, Fairfax, VA \\ 22030-4444, USA
}

\begin{abstract}
We analyze recent results of atomistic computer simulations of grain boundary (GB) diffusion in metals. At temperatures well below the bulk melting point $T_{m}$ GB diffusion occurs by random walk of individual vacancies and self-interstitials. Both defects are equal participants in the diffusion process and can move by a large variety of diffusion mechanisms, many of which are collective transitions. GB diffusion coefficients can be computed by kinetic Monte Carlo simulations. At high temperatures, the presence of large concentrations of point defects is likely to alter the diffusion mechanisms. Molecular dynamics simulations of GB structure and diffusion in copper reveal a continuous GB premelting in close vicinity of $T_{m}$. However, diffusion in high-energy GBs becomes almost independent of the GB structure ("universal") at temperatures well below $T_{m}$. This behavior can be tentatively explained in terms of heterophase fluctuations from the solid to the liquid phase. The exact diffusion mechanisms in the presence of heterophase fluctuations are yet to be established. (c) 2005 Springer Science + Business Media, Inc.
\end{abstract}

\section{Introduction}

Atoms in grain boundaries (GBs) are known to be much more mobile than in the lattice [1]. For example, in fcc metals the difference between the respective diffusion coefficients is about 10 orders of magnitude at about half of the melting temperature. Given this huge difference between the diffusivities it is not surprising that GB diffusion controls a variety of processes in materials, including many phase transformations, some modes of plastic deformation and fracture, creep, etc. [1]. Another important aspect is that GB diffusion is highly sensitive to GB structure. This makes GB diffusion measurements, as well as calculations, an efficient tool for probing the structural state of GBs. While a large amount of experimental data has been measured over the years [1], atomic mechanisms of GB diffusion are still not completely understood and continue to be an attractive subject of research. Some of the questions that still need to be answered include:

1. Which point defects dominate thermal disorder in GBs?

2. What are the atomic mechanisms of GB diffusion?

3. What is the relation between GB diffusion and GB structure, energy, and other GB properties?

4. How can we predict GB diffusion coefficients by atomistic calculations?

The first two questions are obviously the most basic ones. The prevailing paradigm of the 1970s-1980s was that there was no fundamental difference between GB diffusion and lattice diffusion. Vacancies were be- lieved to be the main defects in GBs, which moved by exchanges with single atoms. From this point of view, the only difference between GB diffusion and lattice diffusion is that the vacancy formation and migration energies in GBs are lower than in the bulk [2]. In the 1990s, this simple picture had to be revised based on atomistic computer simulations [3-11]. The goal of this paper is to review the current status of the field with emphasis on our recent work. The point which we wish to emphasize here is that $G B$ diffusion is profoundly different from lattice diffusion. The real picture of GB diffusion is much more complex and is characterized by a large multiplicity of diffusion mechanisms.

In Section 2 we provide a short overview of our recent studies of GB diffusion at low temperatures. We start by reviewing the relevant methodology, followed by a discussion of its applications to atomistic simulations of point defects and diffusion in copper GBs. In Section 3 we present our new results on GB diffusion at high temperatures, including temperatures close to the bulk melting point $T_{m}$. Finally, in Section 4 we briefly summarize and outline future work.

\section{Grain boundary diffusion at low temperatures}

2.1. Methodology

Since we are interested in metals, atomic interactions are modeled with semi-empirical many-body potentials based on the embedded-atom method (EAM) [12]. The potentials are constructed by fitting to both experimental and first-principles data [13-16]. GBs are created by standard geometric constructions and their ground state 
structures are determined by molecular static calculations including both local atomic displacements and rigid translations of the grains $[9,10]$. To understand diffusion mechanisms in a particular GB, we first study point defects in it. Either a vacancy or a self-interstitial is created at various positions in the GB core and the defect free energy is evaluated using molecular statics and the harmonic approximation to atomic vibrations. Knowing the free energies of the point defects, their occupation probabilities at all locations within the GB core are determined. This information already allows us to identify the type of defect that is most abundant in the given GB.

To study diffusion mechanisms, a single point defect is created in the GB core and its walk along the GB is simulated by running molecular dynamics (MD). During the MD run, all defect jumps are detected automatically and the relevant snapshots are saved for a subsequent analysis. By carefully analyzing the snapshots, the most typical diffusive events, or mechanisms, induced by the defect are identified. Although such mechanisms are often referred to as "defect jumps", they can actually represent complex atomic transitions involving a simultaneous displacement of several atoms. Next, absolute transition rates of the defect jumps are calculated using the harmonic transition state theory [17]. The saddle points of the jumps, which are required for this calculation, are determined by the nudged elastic band method $[18,19]$.

Further calculations can proceed along two different lines. The defect jump rates can be compiled into a rate catalog and the latter can be used as input for kinetic Monte Carlo (KMC) simulations [8, 9]. Combined with known defect occupation probabilities, KMC simulations allow accurate calculations of diffusion coefficients within the GB, as well as the jump correlation factors and other kinetic characteristics. Such calculations are performed for vacancies and interstitials separately. The GB diffusion coefficients are determined for different directions within the GB core. Thus, the type of point defect and its particular jumps that dominate diffusion in the GB can be identified. Although such calculations can cover a wide temperature range, it should be emphasized that the results are only valid at relatively low temperatures where diffusion occurs by the motion of single point defects. At high temperatures, interactions between defects can modify their jump rates and even give rise to new diffusion mechanisms involving defect complexes. In fact, at high enough temperatures the very notion of a point defect may loose its significance. It should also be mentioned that, even at low temperatures, the reliability of KMC simulations depends on the size of the rate catalog, which can never comprise all possible diffusive events.

Alternatively, the diffusion coefficients can be determined directly by MD simulations [4, 11]. Namely, the GB is first brought to point-defect equilibrium by a long MD run, followed by another run in which the meansquared displacements of atoms within the GB core are computed. The diffusion coefficient is then determined from the Einstein relation. Here again, the diffusion coefficient can be calculated for any direction within the GB core. Such calculations are free from the model assumptions inherent in KMC simulations. However, to accumulate an adequate statistics of atomic jumps, MD simulations should be implemented at high enough temperatures, usually above $0.6-0.7 T_{m}$.

This methodology has been applied to a series of $\mathrm{Cu}$ GBs [9-11]. The EAM potential developed in [14] was used through this work. Point-defect properties were studied in over a dozen of symmetrical tilt GBs with $\Sigma$ values ranging from 3 to 37 and with three different tilt axes: [100], [110] and [111] ( $\Sigma$ being the reciprocal density of coincident sites). These boundaries represent a sample of typical GBs in fcc metals. In 7 of these GBs, the diffusion coefficients were computed by KMC simulations [9] and in three of them by MD simulations (Section 3 of this paper). Some of the calculations were repeated for $\mathrm{GBs}$ in $\mathrm{Al}$ and $\mathrm{Ni}$, with the results being qualitatively similar to those for $\mathrm{Cu}$. We thus believe that most of our conclusions are generic.

\subsection{Point defects in grain boundaries}

In agreement with earlier work, the vacancy formation energy in GBs, $E_{v}$, has been found to be on average lower than in the bulk. However, variations of $E_{v}$ from site to site within the GB core are tremendous. $E_{v}$ can be as low as $10 \%$ of its bulk value at some sites and above the bulk value at other sites. These variations can be linked to the existence of alternating regions of tension and compression in the GB core $[10,11]$. The same is true for self-interstitials, whose formation energy in GBs is also on average lower than in the lattice but shows large variations between sites. The interaction of GBs with point defects is short-ranged, i.e., limited to distances comparable to the lattice spacing.

There is a distinct correlation between the lowest vacancy formation energy in a GB and the GB energy $\gamma$. Despite a scatter of data points from one GB to another, there is a clear trend for the lowest vacancy formation energy to decrease with GB energy [9-11]. An extrapolation of this correlation to $E_{v} \rightarrow 0$ gives us an estimate of the highest possible energy of stable GBs in the material. Indeed, if $E_{v}$ is negative at least at one site within the GB core, the GB structure is unstable against vacancy formation and should transform to a new structure. A similar correlation has been found for GB interstitials, the respective upper limit of stable GB energies, $\gamma_{\max }$, being approximately the same as for vacancies. For example, in $\mathrm{Cu} \gamma_{\max } \approx 1 \mathrm{~J} / \mathrm{m}^{2}$.

Another important observation is that the vacancy and interstitial formation energies in GBs are close to one another. Thus, in contrast to the common view both defects are equally important in GBs. Neglecting GB interstitials in theories or calculations is not justified.

A variety of structural forms of vacancies and interstitials has been found in GBs. Lattice vacancies are known to be well localized at lattice sites while interstitials form split dumbbell configurations. GB vacancies can also be localized at certain sites. Often, however, atomic relaxations around a vacancy are so strong that it becomes effectively delocalized over a relatively large region. Sometimes, the same delocalized 
configuration can result from removing atoms from different (but of course nearby) sites. Thus, by examining such a delocalized configuration one can never tell where the vacancy was initially created. The vacancy delocalization effect has been found in many GBs.

Vacancy instability is another interesting effect found in GBs. When an atom is removed from a site 1 to create a vacancy, the vacant site gets filled by a neighboring atom 2 during the subsequent static relaxation. As a result, we end up with a vacancy at site 2 and not at the intended site 1 . In other words, site 1 does not support a stable vacancy. As the vacancy delocalization, the vacancy instability effect is very common in GBs. In fact, in some of the GBs studied in this work, less than half of the GB sites are able to support a stable vacancy [9-11].

GB interstitials can exist in three structural forms. Firstly, they can be localized in a relatively open space ("pore") in the GB structure. In the lattice, this structural form is common to interstitial impurity atoms but is usually unstable for self-interstitials. Secondly, GB interstitials can form split dumbbell configurations with various orientations, similarly to lattice interstitials. Finally, on a few occasions highly delocalized GB interstitials have been observed. Their structure can be similar to a crowdion aligned parallel to the tilt axis, or can represent an almost isotropic region with strong static atomic displacements.

\subsection{Diffusion mechanisms in grain boundaries}

The multiplicity of structural forms of point defects in GBs gives rise to a large variety of their diffusion mechanisms. Vacancies often move in GBs by exchanges with individual atoms, just as they do in the lattice. However, they can also induce collective jumps of atoms, which are also called "long vacancy jumps" [9]. Such jumps typically involve two or three atoms. A typical scenario of a long vacancy jump is as follows. A vacancy is trying to make a jump to a neighboring site where it is unstable. Since this is impossible, a group of two or three atoms jumps simultaneously and the vacancy ends up at a different site where it is stable. Thus, long vacancy jumps essentially arise from the existence of unstable vacancies. Not surprisingly, GBs with a large number of unstable vacancy sites are dominated by long vacancy jumps [9]. Such collective jumps should not be confused with so-called dynamic correlations, in which a vacancy makes a new jump before it has a chance to thermalize after a previous jump. In our case, a collective jump has a unique and well-defined saddle point and follows the transition state theory.

Interstitial atoms can move by direct jumps, i.e. by hopping between interstitial positions, or by indirect jumps that involve a simultaneous displacement of several atoms. Typically, an interstitial atom kicks out a nearby regular atom into an interstitial position in a neighboring structural unit and takes its place. Alternatively, the displaced atom, in turn, kicks out its neighbor and the latter becomes an interstitial atom, a process which involves three atoms. Interstitial transitions involving up to four atoms have been found in some GBs.
Under this mechanism, an average atom spends some time in an interstitial position and some time in a regular GB position. As with long vacancy jumps, we again have a group of atoms jumping in a concerted manner.

Another interesting group of mechanisms are ring processes in which a group of atoms implements a collective displacement in a cyclic manner [8]. Ring mechanisms can be induced by point defects or can happen spontaneously without any pre-existing defects. In contrast to the vacancy and interstitial mechanisms which can also operate in the lattice, ring processes have never been found in lattice diffusion.

Having a picture of diffusion mechanisms that operate in a particular $\mathrm{GB}$, a rate catalog is constructed and the diffusion coefficients are determined by KMC simulations. We emphasize again that such calculations are only accurate at low temperatures. An extrapolation of the diffusion coefficients to high temperatures is not justified for a number of reasons: lattice vibrations at high temperatures are not exactly harmonic, point defects can interact with each other and give rise to new diffusion mechanisms, and even the GB structure itself may alter with temperature. However, the low-temperature results are reliable and can be summarized as follows:

1. Despite the involvement of many diffusive events with different activation energies, the GB diffusion coefficients follow the Arrhenius Law quite accurately, suggesting that diffusion is actually dominated by one type of jump or perhaps a group of jumps with nearby activation energies.

2. There is no unique mechanism of GB diffusion: either vacancies or interstitials can dominate the diffusion flux, depending on the GB structure, temperature, and even the diffusion direction.

3. The anisotropy of GB diffusion can be very significant and tends to increase as the temperature is decreased. Diffusion parallel to the tilt axis is not necessarily faster than diffusion normal to it as is often assumed.

4. Depending on the GB structure, GB diffusion coefficients at a given temperature can vary over several orders of magnitude. Thus, the "average" GB diffusivity in a polycrystalline material is not a well defined physical quantity.

As indicated above, the multiplicity of point defect formation energies and structures can be explained by the existence of large internal stresses and stress gradients in GBs. Such stresses have been found to change abruptly from one GB site to another in both magnitude and sign [10]. The stress gradients can strongly bias the vacancy formation energy at neighboring sites (say, 1 and 2) as depicted schematically in Fig. 1. As a result, the barrier of the vacancy jump decreases and at some point can turn to zero. Then, the vacancy becomes unstable with respect to a spontaneous jump from site 1 to site 2 , an effect which is manifested in the vacancy instability observed in many GBs. In a transient situation, the barrier can be very small but not quite zero. Then, the vacancy can be considered to be halfway from site 1 to site 2 . This loosely stable state is accompanied by 

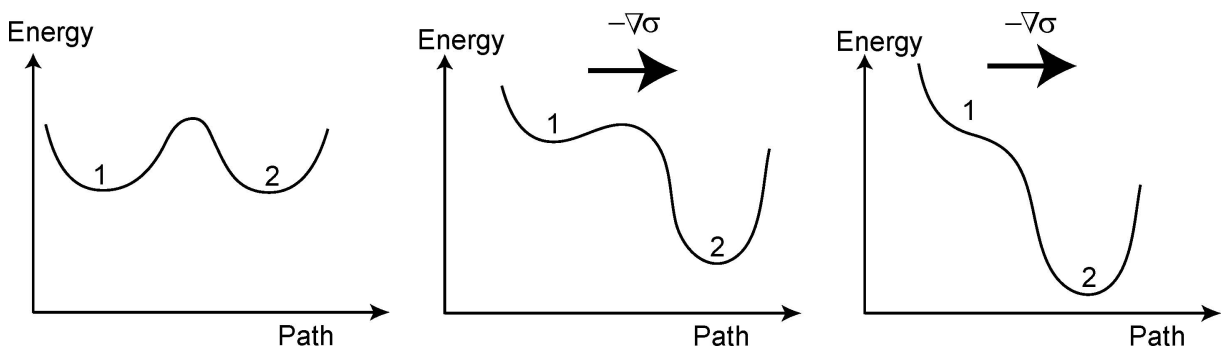

Figure 1 Schematic illustration of the effect of mechanical stresses $(\sigma)$ on the formation energy and jump barrier of point defects in grain boundaries. 1 and 2 are equilibrium positions of a defect. When the jump barrier vanishes, the defect becomes unstable. When the barrier is very low, the defect is delocalized.

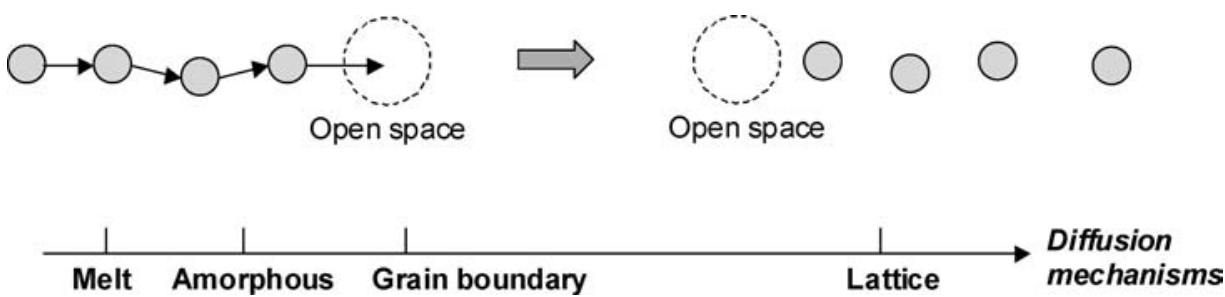

Figure 2 Schematic illustration of a typical diffusion mechanism in grain boundaries. The arrows show atomic dislacements. The scale symbolizes the placement of grain boundaries in terms of their diffusion mechanisms closer to amorphous solids than to regular lattice.

large atomic relaxations and corresponds to a delocalized vacancy. These considerations can be extended to delocalized interstitials. Furthermore, according to this model large enough external stresses can concentrate in some locations within the GB core, destabilize or delocalize point defects, and even trigger new diffusion mechanisms.

Returning to the question of whether GB diffusion is similar to regular lattice diffusion, the foregoing discussion demonstrates that in GBs, in contrast to the lattice, both vacancies and interstitials exist as equal partners. They have a broad spectrum of formation energies and a variety of structural forms. Both defects can move by a variety of diffusion mechanisms, including collective events. If we had to describe the most typical diffusive event in GBs, this would be a collective displacement of an atomic chain in which the head atom fills some open space (free volume) while the tail atom leaves an open space behind (Fig. 2). Thus, in terms of diffusion mechanisms GBs should be placed closer to bulk metallic glasses [20] than to perfect lattice. This parallel with metallic glasses can be linked to the highly nonhomogeneous atomic structure of GBs, which is characterized by a wide spectrum of local environments and tensioncompression gradients, existing despite the periodicity of the structure.

\section{Grain boundary diffusion at high temperatures}

\subsection{Calculation of diffusion coefficients}

At high enough temperatures, GB diffusion coefficients can be computed directly by MD simulations, see e.g. $[4,21]$ and references therein. We have applied this method to calculate diffusion coefficients in three $\mathrm{Cu}$ GBs: $\sum=5(210)[001]$ and $\sum=5(310)[001]$ as representatives of high-energy boundaries $(\gamma=0.952$ and $0.905 \mathrm{~J} / \mathrm{m}^{2}$, respectively), and the $\sum=17(530)[001]$ GB which has a lower energy $\left(\gamma=0.852 \mathrm{~J} / \mathrm{m}^{2}\right)$ and pro- duces a distinct cusp on the orientation dependence of $\gamma$. The boundary conditions were chosen to be periodic in the directions parallel to the GB plane and fixed in the normal direction $[9,10]$. The simulation block contained 9,000-711,000 dynamic atoms and 3,000-5,000 fixed atoms, depending on the particular GB. The fixed layers represented the perfect lattice far away from the GB. They also served to suppress spontaneous GB migration and shear deformation of the block that could otherwise occur at high temperatures. ${ }^{1}$ Prior to MD simulations, the block was expanded uniformly by the thermal expansion factor at the intended temperature, which had been determined separately by zero-pressure Monte Carlo simulations [14]. The NVT ensemble was simulated using a Nose-Hoover thermostat. Prior to diffusion calculations, the block was "pre-annealed" by running MD for up to $1 \mathrm{~ns}$ to allow for the generation of equilibrium point defects. The time of the "diffusion anneal" varied between 1 and $10 \mathrm{~ns}$, depending on the temperature. The diffusion coefficient $D$ was computed by linearizing a plot of $\left\langle X^{2}\right\rangle$ versus $t$ and using the Einstein relation $D=\left\langle X^{2}\right\rangle / 2 t$. Here, $t$ is the diffusion time and $\left\langle X^{2}\right\rangle$ is the mean-squared displacement, in a particular direction $X$, of atoms contained within a window of $\pm d$ around the GB. Since we were interested in diffusion within the GB core, $d$ was chosen to be $0.5 \mathrm{~nm}$ throughout this work. This choice is somewhat arbitrary but it was found that variations in $d$ only produced almost uniform shifts of all diffusion coefficients without affecting our main conclusions. At high temperatures, the GBs were observed to slightly move up and down around a medium position. In some

\footnotetext{
${ }^{1}$ There is coupling between GB migration and relative tangential displacement of grains [22-24]. Free-surface boundary conditions readily accommodate grain translations and thus facilitate spontaneous GB migration around an equilibrium position. Fixed layers prevent grain translations and effectively suppress GB motion, although do not eliminate it completely.
} 


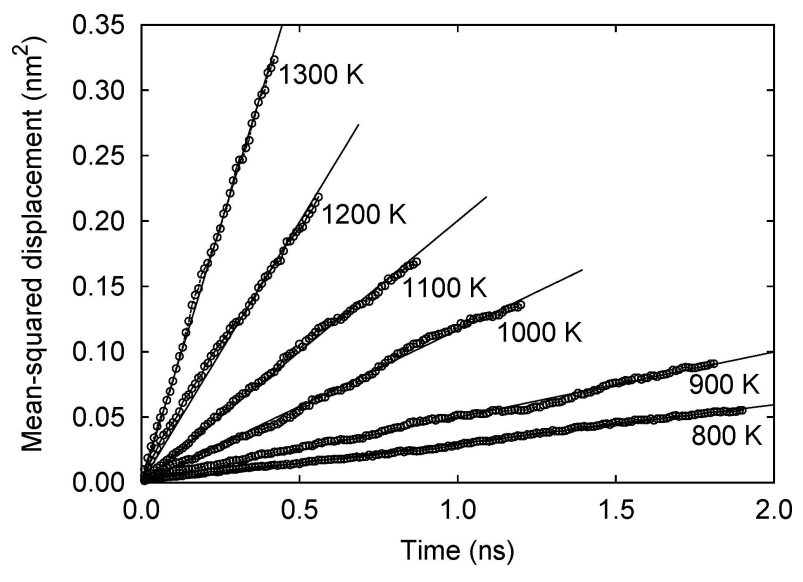

Figure 3 Typical plots of the mean-squared atomic displacement in the core versus diffusion time obtained at different temperatures by molecular dynamic simulations of the $\Sigma=5(210)$ [001] grain boundary in copper. The slopes of the plots determine the diffusion coefficients.

cases, the GB was observed to leave the diffusion window $( \pm d)$, in which case the diffusion simulation had to be stopped. In fact, it was this random motion of the GBs that limited the diffusion simulation time at high temperatures. The time of low-temperature simulations was limited by our computational resources. Fig. 3 illustrates typical $\left\langle X^{2}\right\rangle$ versus $t$ plots obtained for the $\sum=5(210)$ GB. Diffusion coefficients in the liquid phase were also calculated using a similar methodology. The bulk melting temperature of $\mathrm{Cu}$ with this EAM potential, $T_{m}=1327 \mathrm{~K}$, was obtained by the interface velocity method after averaging over three different interface orientations $\left(T_{m}=1358 \mathrm{~K}\right.$ in experiment).

The diffusion coefficients obtained are summarized in the Arrhenius diagram in Fig. 4. For comparison, we include the experimental GB diffusion coefficients obtained by radiotracer measurements on polycrystalline

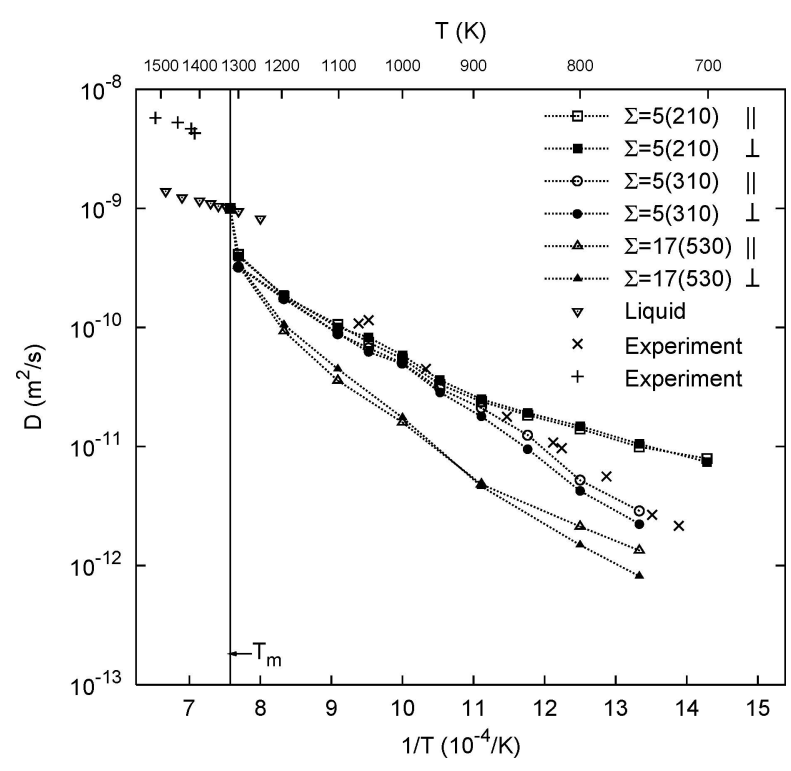

Figure 4 Arrhenius diagram of diffusion coefficients $(D)$ parallel (||) and perpendicular $(\perp)$ to the tilt axis in three $\mathrm{Cu}$ grain boundaries and in liquid $\mathrm{Cu}$. Obtained by molecular dynamics simulations. Experimental data: $\times$ - grain boundary diffusion in polycrystalline $\mathrm{Cu}[25]$; + diffusion in liquid $\mathrm{Cu}$ [26]. The dashed lines serve as a guide of the eye.
$\mathrm{Cu}$ samples [25]. ${ }^{2}$ This comparison demonstrates that the order of magnitude of the calculated diffusion coefficients is very reasonable. The calculated liquid diffusion coefficients are also reasonable but lie on the lower side of experiment [26]. Diffusion in the $\sum=17 \mathrm{~GB}$ is slower than in the $\sum=5 \mathrm{GBs}$ at all temperatures, which is consistent with the expected correlation between GB diffusion and GB energy [9,27-29]. We also observe that about $50-100 \mathrm{~K}$ before the bulk melting temperature GB diffusion accelerates, diffusion coefficients in different GBs converge to the same value, and that value continuously approaches the diffusion coefficient in the liquid phase at $T=T_{m}$. This behavior is in agreement with previous atomistic simulations which revealed a continuous premelting at GBs in a close vicinity of $T_{m}$ [22,30-34].

Another interesting effect revealed by Fig. 4 is that the diffusion coefficients in the two $\sum=5$ GBs merge together about $300-400 \mathrm{~K}$ below $T_{m}$. A similar effect was found earlier by Keblinski et al. [21] in palladium GBs. The merger of the diffusion coefficients may suggest that GB diffusion becomes structure-independent, or "universal" [21]. Keblinski et al. [21] suggested that high-energy GBs in metals develop a "liquid-like" structure well below the melting point and that this structure gives rise to "liquid-like" diffusion mechanisms. Therefore, we have carefully studied the GB structures in this temperature range.

\subsection{Grain boundary structure at high temperatures}

By examining multiple MD snapshots it was found that, although the atomic disorder in the GBs increased with temperature, individual structural units characterizing the ordered GB structure could still be identified up to a few tens of degrees below $T_{m}$. Dynamic steps could also be noticed in the GBs. The GB width tended to gradually increase with temperature while always remaining on the order of a nanometer until very close to $T_{m}$. Near the melting point, the recognizable structural units were typically separated by regions of strong atomic disorder. This picture, however, was fully dynamic: the disordered regions observed in one snapshot could be found ordered in the next one and vise versa. Overall, there was no indication of the GBs turning into permanent liquid layers below $T_{m}$.

To quantify these observations, we have followed [35] to compute the layered static structure factor in the GBs at various temperatures. Namely, the static structure factor $|S(\mathbf{k})|^{2}$ relating to layers parallel to the GB plane was calculated with the wave vector of $\mathbf{k}=[0,0,4 \pi / a]$ ( $a$ being the lattice parameter). As in [22], atomic positions averaged over 1 ps time intervals were used for the $|S(\mathbf{k})|^{2}$ calculations, which eliminated much of the scatter associated with atomic vibrations. The calculation was repeated 20-30 times over a long MD run and the results were averaged to

\footnotetext{
${ }^{2}$ The quantity measured in experiments is the product $D \delta, D$ being the GB diffusion coefficient and $\delta$ the GB diffusion width. We determined $D$ assuming $\delta=1 \mathrm{~nm}$.
} 


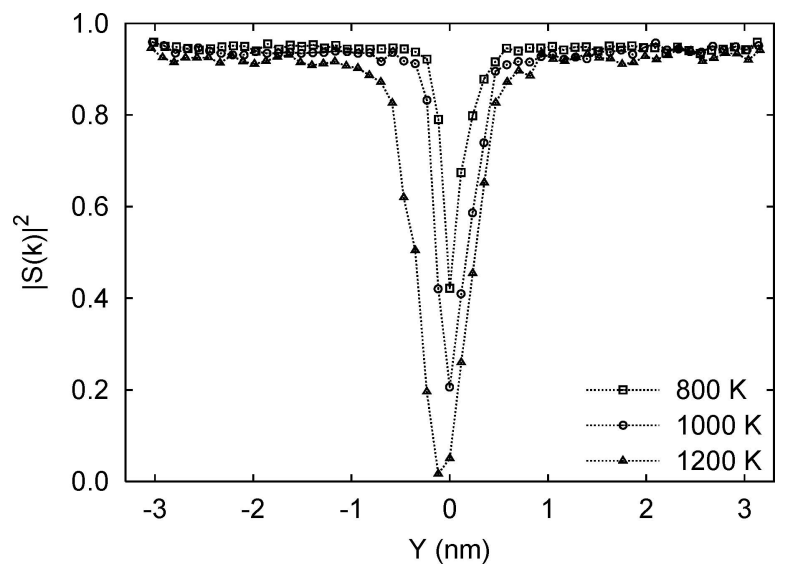

Figure 5 Static structure factor as a function of distance $Y$ across the $\Sigma=5(310)$ grain boundary in $\mathrm{Cu}$ at selected temperatures. $Y=0$ corresponds to the boundary position at $T=0$. The long-range order in the boundary core decreases with temperature while the boundary width increases. Notice the small displacement of the boundary to the left at $1200 \mathrm{~K}$.

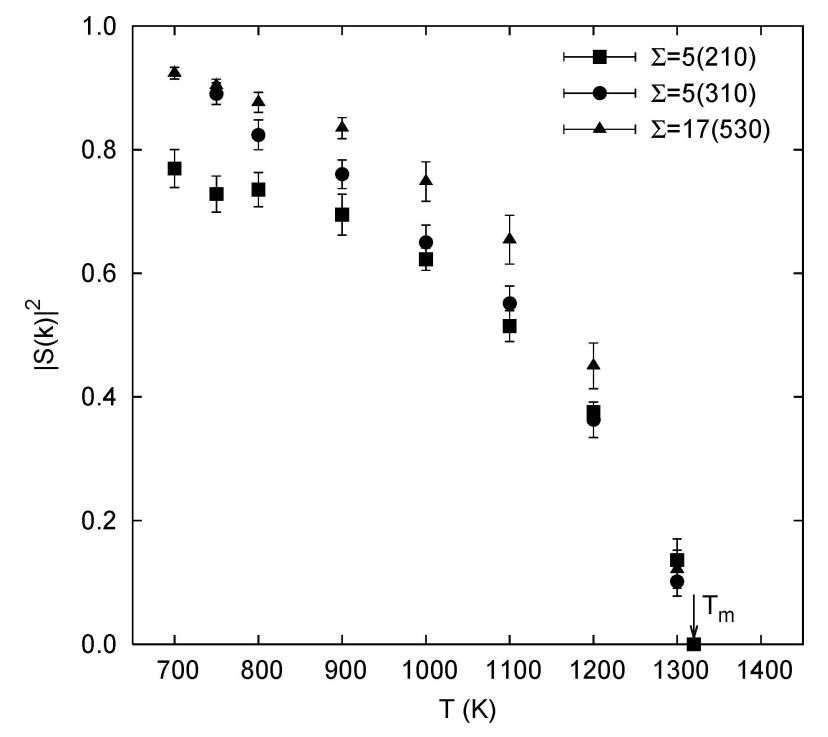

Figure 6 Static structure factor within the grain boundary core as a function of temperature for three grain boundaries in $\mathrm{Cu}$.

produce a profile of $|S(\mathbf{k})|^{2}$ across the GB. Typical profiles displayed in Fig. 5 demonstrate that the long-range order in the GB core decreases with temperature. At the same time the GB thickness, defined by the width of the minimum of $|S(\mathbf{k})|^{2}$, increases and eventually diverges at $T \rightarrow T_{m}$. To quantify the average degree of long-range order within the GB core, $|S(\mathbf{k})|^{2}$ was averaged over a region $\pm d$ around the GB. The core half-thickness $d$ was chosen to be the same as in the diffusion calculations, $d=0.5 \mathrm{~nm}$, which enabled us to examine correlations between long-range order and GB diffusion. ${ }^{3}$

Fig. 6 shows that $|S(\mathbf{k})|^{2}$ in the GB core is a continuous function of temperature below $T_{m}$. This function remains well positive at all temperatures except very close to $T_{m}$. It decreases rapidly near $T_{m}$ and turns to zero exactly at $T=T_{m}$. This behavior is well consistent with the similar behavior of the GB diffusion

${ }^{3}$ Different choices of $d$ resulted in simultaneous shifts of both diffusion coefficients and structure factors without altering the basic trends. coefficients (cf. Fig. 4) and is compatible with the idea of continuous premelting in a close proximity of $T_{m}$. Based on the existence of long-range order in the GBs we can rule out the formation of permanent liquid layers below $T_{m}$. We note also that the faster diffusion in the $\sum=5 \mathrm{GBs}$ in comparison with the $\sum=17 \mathrm{~GB}$ (Fig. 4) correlates with the consistently lower degree of ordering in the $\sum=5$ GBs (Fig. 6). Furthermore, observe that the structure factors in the two $\sum=5 \mathrm{GBs}$ approach each other well below $T_{m}$, as do the respective GB diffusion coefficients. This again points to a possible "universal" structure and diffusion mechanisms in these GBs above $900 \mathrm{~K}$. Unfortunately, we have not been able to identify the relevant mechanisms based on our MD simulations. The analysis of dynamic trajectories of atoms has so far not been successful because of massive atomic displacements accompanying the diffusion process.

As a working model, it is reasonable to consider GB premelting as a dynamic process [21] and apply the concept of heterophase fluctuations to describe it [36-40]. According to this model, at high enough temperatures a GB can undergo local melting events as fluctuations to the liquid phase. Each small embryo of liquid exists for a short time (perhaps $\sim 10 \mathrm{ps}$ ) before it crystallizes back to the ordered GB structure. Because such events are local, long-range order can still exist. The atomic displacements accompanying the fluctuations can constitute the dominant mechanism of GB diffusion, which in this case would depend more on diffusive properties of the liquid phase rather than on details of the GB structure ("universal" mechanism). The free-energy barrier $\Delta G$ that needs to be overcome for a fluctuation is lower in high-energy GBs, such as the $\sum=5$ 's, and can be prohibitively large in low-energy GBs. This can explain the greater role played by the heterophase fluctuations in high-energy GBs. Regardless of the GB energy, however, $\Delta G \rightarrow 0$ at $T \rightarrow T_{m}$. Therefore, the amount of heterophase fluctuations in any GB should drastically increase at $T \rightarrow T_{m}$ and finally lead to its transformation into a thick layer of real liquid at $T=T_{m}$.

\section{Summary}

GB diffusion is a complex process that can occur by a variety of atomic mechanisms. Many of such mechanisms are collective transitions involving either vacancies or interstitials. Vacancies and interstitials are present in GBs in comparable amounts and can exist in a variety of structural forms, some of which are delocalized. Some GB sites are unable to support a stable vacancy. At relatively low temperatures, GB diffusion is mediated by the motion of individual point defects. Under such conditions, GB diffusion coefficients can be readily calculated by catalog-based KMC simulations with transition rates computed within the harmonic transition state theory. One or a few diffusive events with nearby activation energies usually dominate the overall diffusion process, with the result that the GB diffusion coefficient closely follows the Arrhenius law. There is no unique mechanism of GB diffusion. The point defect that dominates GB diffusion depends on the particular GB structure, temperature and even the 
diffusion direction. Overall, the atomic picture of GB diffusion is quite far from that of lattice diffusion and is probably closer to diffusion in metallic glasses.

At high temperatures, point defect concentrations in GBs become so high that the model of isolated defects no longer applies. Defect-defect interactions begin to play a crucial role and can give rise to new diffusion mechanisms. Under such conditions, MD simulations present the most adequate tool for diffusion calculations. Such calculations show that GB diffusion continues to follow the Arrhenius $\mathrm{Law}^{4}$ until very close $(\sim 50 \mathrm{~K})$ to the bulk melting point $T_{m}$. In the latter region, it accelerates and continuously approaches diffusion in the liquid phase. Both our and previous [21] MD simulations show that diffusion in some high-energy GBs can become "universal", i.e., independent of the particular GB structure. Analysis of high-temperature GB structures shows that they do not develop permanent liquid layers and maintain some degree of long-range order until very close to $T_{m}$. The degree of long-range order, as well as the GB width, diffusivity and other characteristics are continuous functions of temperature. In other words, our simulations give no indication of premelting as a GB phase transformation happening at a particular temperature below $T_{m}$. Tentatively, GB structure and diffusion at high temperatures can be explained in terms of heterophase fluctuations from the solid to the liquid phase. However, this model is only conceptual and requires further quantitative analysis in the future. Exact atomic mechanisms of GB diffusion in the presence of heterophase fluctuations are not yet known and should also be the subject of future research.

\section{Acknowledgements}

This work was supported by the U.S. Department of Energy (Office of Basic Energy Sciences) through grant No. DE-FG02-01ER45871.

\section{References}

1. I. KAUR, Y. MISHIN and W. GUST, in Fundamentals of Grain and Interphase Boundary Diffusion, (Wiley, Chichester, West Sussex, 1995).

2. R. W. B ALLUFFI, in Diffusion in Crystalline Solids," edited by G. E. Murch and A. S. Nowick (Academic Press, New York, 1984) p. 319.

3. Q. MA, C. L. LIU, J. B. ADAMS and R. W. BALLUFFi, Acta metall. mater. 41 (1993) 143.

4. C. L. LIU and S. J. Plimpton, Phys. Rev. B 51 (1995) 4523.

\footnotetext{
${ }^{4}$ Arrhenius parameters may alter with temperature, signifying changes in the diffusion mechanism. For example, the activation energies of diffusion in the $\Sigma=5(210)$ and $\Sigma=5(310)$ GBs are different at temperatures $T<900 \mathrm{~K}$ but change and become almost identical above $900 \mathrm{~K}$ (Fig. 4).
}

5. M. NOMURA, S.-Y. LEE and J. B. ADAMS, J. Mater. Res. 6 (1991) 1.

6. M. NOMURA and J. B. AD A MS, J. Mater. Res. 7 (1992) 3202.

7. Idem, ibid. 10 (1995) 2916.

8. M. R. S $\emptyset$ RENSEN, Y. MISHIN and A. F. VOTER, Phys. Rev. B 62 (2000) 3658.

9. A. SUZUKI and Y. MISHIN, Interf. Sci. 11 (2003) 131.

10. Idem., ibid. 11 (2003) 425.

11. A. SUZUKI and Y. MISHIN, J. Metast. Nonocryst. Mater. 19 (2004) 1.

12. M. S. D AW and M. I. B A S KES, Phys. Rev. B 29 (1984) 6443.

13. Y. MISHIN, D. FARKAS, M. J. MEHL and D. A. PAPACONSTANTOPOULOS, ibid. 59 (1999) 3393.

14. Y. MISHIN, M. J. MEHL, D. A. PAPACONSTANTOPOULOS, A. F. VOTER and J. D. KRES S, Phys. Rev. B 63 (2001) 224106.

15. Idem., ibid. 65 (2002) 224114.

16. Y. MISHIN, Acta Mater. 52 (2004) 1451

17. G. H. VINEYARD, Phys. Chem. Solid 3 (1957) 121

18. H. JÓ NSSON, G. MILLS and K. W. JACOBSEN, in: "Classical and Quantum Dynamics in Condensed Phase Simulations," edited by B. J. Berne, G. Ciccotti and D. F. Coker, (World Scientific, Singapore, 1998).

19. G. HENKELMAN, G. JOHANNESSON and H. J Ó NSSON, in "Theoretical Methods in Condensed Phase Chemistry," edited by S. D. Schwartz of Progress in Theoretical Chemistry and Physics, Chapt. 10 (Kluwer Academic Publishers, 2000) Vol. 5.

20. A. HEESEMANN, V. ZOLLMER, K. RATZKE and F. FAUPEL, Phys. Rev. Lett. 84 (2000) 1467.

21. P. KEBLINSKI, D. WOLF, S. R. PHILlpOT and H. GLEITER, Philos. Mag. A 79 (1999) 2735.

22. T. NGUYEN, P. S. HO, T. KWOK, C. NITTA and S. YIP, Phys. Rev. B 46 (1992) 6050.

23. J. W. CAHN and J. E. TA Y L OR, Acta Mater. 52 (2004) 4887.

24. A. SUZUKI and Y. MISHIN, to be published.

25. T. SURHOLT and CHR. HERZIG, Acta Mater. 45 (1997) 3817.

26. J. HENDERSON and L. YANG, Trans. AIME 221 (1961) 72.

27. V. T. BORISOV, V. M. GOLIKOV and G. V. SCHER B EDIN S K Y, Phys. Met. Metallogr. 17 (1964) 80.

28. D. GUPT A, Metall. Trans. A 8 (1977) 1431.

29. Idem., Interf. Sci. 11 (2003) 7.

30. G. CICCOTTI, M. GUILlope and V. PONTIKIS, Phys. Rev. B 27 (1983) 5576.

31. M. GUillope, G. CiCcotti and V. POntikis, Surf. Sci. 144 (1984) 67.

32. J. Q. BROUGHTON and G. H. GILMER, Phys. Rev. Lett. 56 (1986) 2692.

33. J. LU and J. A. SZPUNAR, Interf. Sci. 3 (1995) 143.

34. J. Q. BROUGHTON and G. H. GIlmer, Modell. Simul. Mater. Sci. Eng. 6 (1998) 87.

35. J. F. LUTSCO, D. WOLF, S. YIP, S. R. PHILlPOT and T. NGUYEN, Phys. Rev. B 38 (1988) 11572.

36. J. FRENKEL, in "Kinetic Theory of Liquids," (Dover, New York, 1955).

37. N. F. МОт T, Proc. Phys. Soc. 60 (1948) 391.

38. A. R. UBBELOHDE, "Molten State of Matter: Melting and Crystal Structure," (Wiley, Chichester, 1978).

39. Y. M. MISHIN and I. M. R AZU MOV S K II, Phys. Status Solidi (a) 117 (1990) 91.

40. Y. M. MiShin and I. M. RAZUMOVSKiI, Acta Metall. Mater. 40 (1992) 2707.

Received 13 September 2004

and accepted 31 January 2005 